\title{
LONGITUDES OF A LINK AND PRINCIPALITY OF AN ALEXANDER IDEAL
}

\author{
JONATHAN A. HILLMAN
}

\begin{abstract}
In this note it is shown that the longitudes of a $\mu$-component homology boundary link $L$ are in the second commutator subgroup $G^{\prime \prime}$ of the link group $G$ if and only if the $\mu$ th Alexander ideal $\mathcal{E}_{\mu}(L)$ is principal, generalizing the result announced for $\mu=2$ by R. H. Crowell and E. H. Brown. These two properties were separately hypothesized as characterizations of boundary links by R. H. Fox and N. F. Smythe.
\end{abstract}

For a $\mu$-component homology boundary link $L$ the first nonvanishing Alexander ideal is $\varepsilon_{\mu}(L)$. If $L$ is actually a boundary link, then $\varepsilon_{\mu}(L)$ is principal and the longitudes of $L$ lie in the second commutator subgroup of the link group [2], [6]. R. H. Crowell and E. H. Brown have announced that the latter two assertions are equivalent for a 2-component homology boundary link [2]. This note presents a proof of the following generalization.

THEOREM. Let $L: \cup_{i=1}^{\mu} S_{i}{ }^{1} \rightarrow S^{3}$ be a (locally flat) $\mu$-component homology boundary link, with group $G$. Then $\mathcal{E}_{\mu}(L)=\left(\Delta_{\mu}\right) \cdot A$ where $A$ is contained in the annihilator ideal (in

$$
\left.\Lambda=\mathbf{Z}\left[\mathbf{Z}^{\mu}\right] \approx \mathbf{Z}\left[t_{1}, t_{1}^{-1}, \ldots, t_{\mu}, t_{\mu}^{-1}\right]\right)
$$

of the image of the longitudes in the $\Lambda$-module $G^{\prime} / G^{\prime \prime}$, and $A$ is contained in no proper principal ideal. Hence $\mathcal{E}_{\mu}(L)$ is principal if and only if the longitudes of $L$ lie in $G^{\prime \prime}$.

Proof. $L$ extends to an imbedding $N: \cup_{i=1}^{\mu} S_{i}^{1} \times D^{2} \rightarrow S^{3}$, since it is locally flat. Let $X=S^{3}-\operatorname{int}(\operatorname{Im}(N))$ have base point $x_{0} \in X-\partial X$. Then $G \approx \pi_{1}\left(X, x_{0}\right)$. Let $p: X^{\prime} \rightarrow X$ be the maximal abelian cover of $X$ and choose $x_{0}^{\prime} \in p^{-1}\left(x_{0}\right)$, so that $\pi_{1}\left(X^{\prime}, x_{0}^{\prime}\right) \approx G^{\prime}$ and $H_{1}\left(X^{\prime}\right)=G^{\prime} / G^{\prime \prime}$. By definition of homology boundary link there is a map

$$
f:\left(X, x_{0}\right) \rightarrow\left(\bigvee_{j=1} S_{j}^{1}, *\right)
$$

inducing an epimorphism of fundamental groups, and $p$ is the pullback via $f$ of the maximal abelian cover of $\bigvee_{j=1}^{\mu} S_{j}^{1}$. Thus $X^{\prime}$ may be constructed by splitting $X$ along "Seifert surfaces", as was done in [3] for boundary links. For

Received by the editors September 12, 1977.

AMS (MOS) subject classifications (1970). Primary 55A25; Secondary 55A10.

Key words and phrases. Homology boundary link, longitude, Seifert surface, maximal abelian covering, Mayer-Vietoris sequence, second commutator subgroup, Alexander ideal, annihilator, principal ideal.

(1) American Mathematical Society 1978 
each $j$ such that $1 \leqslant j \leqslant \mu$, choose $P_{j} \in S_{j}^{1}$ distinct from the wedge-point $*$, and let $V_{j}=f^{-1}\left(P_{j}\right)$. After homotoping $f$ if necessary, each $V_{j}$ may be assumed a connected, bicollared submanifold. Let $Y=X-\cup_{j=1}^{\mu}$ int $W_{j}$, where the $W_{j}$ are disjoint regular neighborhoods of the $V_{j}$ in $X$. There are two natural embeddings of each $V_{j}$ in $Y$; call one $\nu_{j+}$ and the other $\nu_{j-}$. (Making such a choice is equivalent to choosing a local orientation for each $P_{i}$ in $\bigvee_{j=1}^{\mu} S_{j}{ }^{1}$, or choosing orientations for the meridians of $L$.) $Y$ is a deformation retract of $X-V$, where $V=\cup_{j=1}^{\mu} V_{j}$. Then one has

$$
\begin{aligned}
X^{\prime} & =Y \times \mathbf{Z}^{\mu} / \nu_{j+}(w) \times\left\langle n_{1}, \ldots, n_{j}+1, \ldots, n_{\mu}\right\rangle \\
& \sim \nu_{j-}(w) \times\left\langle\dot{n}_{1}, \ldots, n_{j}, \ldots, n_{\mu}\right\rangle, \quad \forall w \in V_{j}, \quad 1 \leqslant j \leqslant \mu .
\end{aligned}
$$

$G^{\prime} / G^{\prime \prime}=H_{1}\left(X^{\prime}\right)$ then appears in the following segment of a Mayer-Vietoris sequence:

$$
\begin{aligned}
H_{1}(V) \otimes \Lambda \stackrel{d_{1}}{\rightarrow} & H_{1}(Y) \otimes \Lambda \rightarrow H_{1}\left(X^{\prime}\right) \\
& \rightarrow H_{0}(V) \otimes \Lambda \stackrel{d_{0}}{\rightarrow} H_{0}(Y) \otimes \Lambda \rightarrow \mathbf{Z} \rightarrow 0
\end{aligned}
$$

where $d_{*} \mid H_{*}\left(V_{j}\right) \otimes \Lambda=\left(\nu_{j+}\right)_{*} \otimes t_{j}-\left(\nu_{j-}\right)_{*} \otimes 1$ and homology is taken with integral coefficients. The map $f$ induces a map from this Mayer-Vietoris sequence to the corresponding one for the maximal abelian covering space of $\bigvee_{j=1}^{\mu} S_{j}^{1}$ :

$$
0-F(\mu)^{\prime} / F(\mu)^{\prime \prime} \rightarrow \Lambda^{\mu} \rightarrow \Lambda \stackrel{\varepsilon}{\rightarrow} \mathbf{Z} \rightarrow 0 .
$$

(Here $F(\mu)$ is the free group of rank $\mu$, and $\varepsilon: \Lambda \rightarrow \mathbf{Z}$ is the augmentation homomorphism.) Since each $V_{j}$ is connected, the maps on the degree zero terms are all isomorphisms. Thus one concludes that

$$
H_{1}(V) \otimes \Lambda \stackrel{d_{1}}{\rightarrow} H_{1}(Y) \otimes \Lambda \rightarrow K \rightarrow 0
$$

is exact, where

$$
K=\operatorname{ker}\left(: G^{\prime} / G^{\prime \prime} \rightarrow F(\mu)^{\prime} / F(\mu)^{\prime \prime}\right)=\operatorname{ker}\left(: H_{1}\left(X^{\prime}\right) \rightarrow H_{0}(V) \otimes \Lambda\right) .
$$

Likewise $f$ induces a map from the 4 term exact sequence of Crowell [1]

$$
0 \rightarrow G^{\prime} / G^{\prime \prime} \rightarrow A(G) \rightarrow \Lambda \stackrel{\varepsilon}{\rightarrow} \mathbf{Z} \rightarrow 0
$$

to the corresponding sequence for $F(\mu)$ (which is just the above MayerVietoris sequence for $\left.\bigvee_{j=1}^{\mu} S_{j}^{1}\right)$ and so $0-K \rightarrow A(G) \rightarrow A(F(\mu))=\Lambda^{\mu} \rightarrow 0$ is exact. From this last short exact sequence one concludes that $\mathscr{E}_{k}(L)=$ $\mathcal{E}_{k}(A(G))$ is equal to the ideal generated by $\cup_{l=0}^{k} \mathcal{E}_{l}(K) \cdot \mathcal{E}_{k-l}\left(\Lambda^{\mu}\right)$; in particular $\mathcal{E}_{\mu-1}(L)=0$ and $\mathcal{E}_{\mu}(L)=\mathcal{E}_{0}(K)$.

Now the $\Lambda$-submodule of $H_{1}\left(X^{\prime}\right)$ generated by the longitudes is the image of $H_{1}\left(\partial X^{\prime}\right)$ via the inclusion map, and is contained in the image of $H_{1}(Y) \otimes$ $\Lambda$, so is contained in $K$. Let $B$ be this submodule, and let $Q$ be the quotient $\Lambda$-module. Thus $0-B-K \rightarrow Q \rightarrow 0$ is exact, and $\mathscr{E}_{0}(K)=\mathcal{E}_{0}(Q) \cdot \mathcal{E}_{0}(B)$ (because $Q$ has a square presentation matrix-see below). It is easy to see that $(\operatorname{Ann}(B))^{\mu} \subset \mathcal{E}_{0}(B):$ if 


$$
\Lambda^{\lambda} \stackrel{M}{\rightarrow} \Lambda^{\mu} \stackrel{\varphi}{\rightarrow} B \rightarrow 0
$$

is a presentation for $B$ with $\varphi\left(e_{i}\right)=e$ th longitude (where $e_{i}$ is the $i$ th standard basis element of $\Lambda^{\mu}$ ), and if $\alpha_{1}, \ldots, \alpha_{\mu} \in \operatorname{Ann}(B)$ then

$$
\Lambda^{\lambda} \oplus \Lambda^{\mu} \rightarrow \Lambda^{\mu} \stackrel{\tilde{M}}{\rightarrow} B \stackrel{\varphi}{\rightarrow} 0
$$

is also a presentation for $B$, where $\tilde{M}=\left(M, \operatorname{diag}\left\{\alpha_{1}, \ldots, \alpha_{\mu}\right\}\right)$, and so

$$
\prod_{i=1}^{\mu} \alpha_{i}=\operatorname{det}\left(\operatorname{diag}\left\{\alpha_{1}, \ldots, \alpha_{\mu}\right\}\right) \in \mathcal{E}_{0}(B) .
$$

It is scarcely more difficult to see that $\varepsilon_{0}(B) \subset \operatorname{Ann}(B)$ : let $\delta$ be the determinant of the $\mu \times \mu$ minor $M^{\prime \prime}$ of $M$. Then

$$
\Lambda^{\mu} \stackrel{M^{\prime \prime}}{\rightarrow} \Lambda^{\mu} \rightarrow \text { Coker } M^{\prime \prime} \rightarrow 0
$$

presents a module of which $B$ is a quotient. Now if $\sum m_{i} e_{i} \in \Lambda^{\mu}$, then by Cramer's rule $\delta \cdot \sum m_{i} e_{i}=M^{\prime \prime}\left(\sum n_{j} e_{j}\right)$ where $n_{j}$ is the determinant at the matrix obtained by replacing the $i$ th column of $M^{\prime \prime}$ with the column of coefficients $\left\{m_{i}\right\}$. Hence $\delta$ annihilates Coker $M^{\prime \prime}$, and a fortiori, $B$. Therefore $\varepsilon_{0}(B)$, which is generated by such determinants, is contained in $\operatorname{Ann}(B)$. Thus to prove the theorem it will suffice to show that $\mathcal{E}_{0}(B)$ is not contained in any proper principal ideal, and that $Q$ has a presentation of the form $\Lambda^{q} \stackrel{P}{\rightarrow} \Lambda^{q} \rightarrow$ $Q \rightarrow 0$ so that $\mathcal{E}_{0}(Q)=(\operatorname{det} P)$ is principal.

Choose base points in $V_{i} \cap \partial N\left(S_{i}^{1} \times D^{2}\right)$ for each $i, 1 \leqslant i \leqslant \mu$, and choose paths from these base points to $\alpha_{0}$. (Equivalently, $X^{\prime}$ contains copies of $V_{i}$ indexed by $\mathbf{Z}^{\mu}$. Choose one such lift, $V_{i}^{\prime}$, for each $i$.) If one now orients the link $L$, the longitudes are unambiguously defined, as elements of $G$. Let $l_{i}$ be the image of the $i$ th longitude in $B$. Since the $i$ th longitude commutes with the $i$ th meridian, one has $\left(t_{i}-1\right) l_{i}=0$. In contrast to the case of boundary links, $\partial V_{j}$ will in general have several components; however $\partial V_{j} \cap \partial N\left(S_{i}^{1} \times\right.$ $\left.D^{2}\right)$ is always homologous in $\partial N\left(S_{i}^{1} \times D^{2}\right)$ to the $i$ th longitude, if $j=i$, and to 0 otherwise. $\partial V_{i}^{\prime}$ is a union of translates of loops in the homology classes $l_{1}, \ldots, l_{\mu}$. Hence there are relations of the form

$$
\sum_{i=1}^{\mu} p_{i j}\left(t_{1}, \ldots, t_{\mu}\right) l_{j}=0
$$

in $B$, and by the above remarks on $\partial V_{j}$, one has $p_{i j}(1, \ldots, 1)=0$ for $i \neq j$ and $p_{i i}(1, \ldots, 1)= \pm 1$. Since $t_{i} \cdot l_{i}=1 \cdot l_{i}$, one may assume that $p_{i}=$ $p_{i i}\left(t_{1}, \ldots, t_{\mu}\right)$ does not involve $t_{i}$. Clearly $p_{i} \prod_{j \neq i}\left(t_{j}-1\right)$ is the determinant of a $\mu \times \mu$ matrix of relations for $B$, and so is in $\varepsilon_{0}(B)$. (For what follows it would be sufficient to observe that it clearly annihilates $B$, and so the $\mu$ th power is in $\varepsilon_{0}(B)$.) Let $(c)$ be a principal ideal containing $\mathcal{E}_{0}(B)$. Since $\Lambda$ is a factorial domain, $c$ may be assumed irreducible. Therefore $p_{1} \Pi_{j>1}\left(t_{j}-1\right) \in$ (c) implies $c$ divides $p_{1}$ or some $\left(t_{j}-1\right)$ for $j>1$. If $c=t_{j}-1$, then $c$ cannot divide $p_{j} \Pi_{k \neq j}\left(t_{k}-1\right)$ which does not involve $t_{j}$. If $c$ divides $p_{i}$ for each $i$, 
$1 \leqslant i \leqslant \mu$, then $c$ involves none of the variables and hence is in $\mathbf{Z}$. Since $p_{i}(1, \ldots, 1)= \pm 1, c= \pm 1$ and so $(c)=\Lambda$.

Let $J=\operatorname{ker}\left(: H_{1}(X-V, \partial X-V) \rightarrow H_{0}(\partial X-V)\right)=H_{1}(X-V) / H_{1}(\partial X$ $-V)$. From the following commutative diagram of $\Lambda$-modules

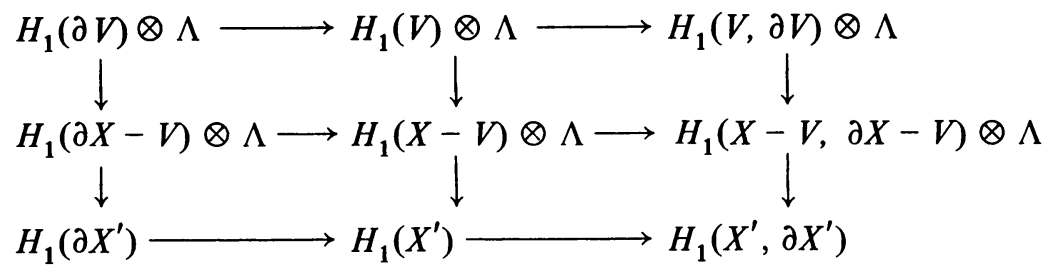

(with rows from exact sequences of pairs and columns from Mayer-Vietoris sequences of $\mathbf{Z}^{\mu}$-covers), one deduces a commutative diagram

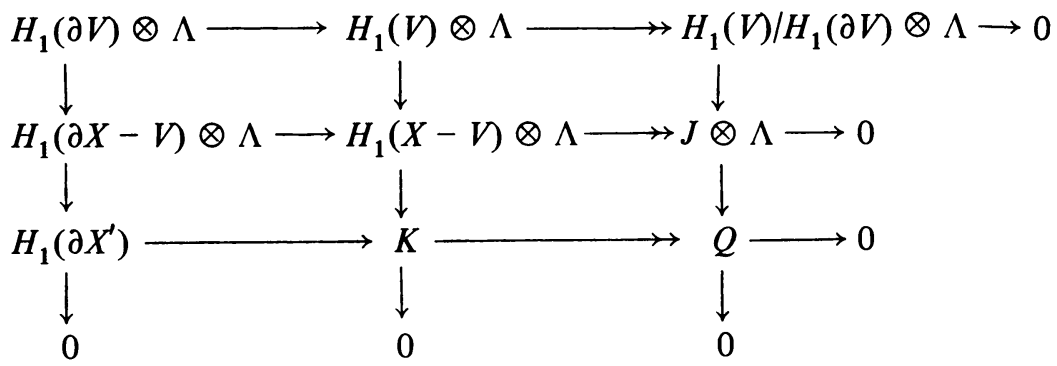

in which all rows and the first two columns are exact. It follows that the third column is exact, and so

$$
\left(H_{1}(V) / H_{1}(\partial V)\right) \otimes \Lambda \rightarrow J \otimes \Lambda \rightarrow Q \rightarrow 0
$$

is a presentation for $Q$. Let $\rho=r k_{\mathrm{z}} H_{1}(V), \sigma=r k_{\mathrm{z}} H_{1}(\partial V)$. Since $0 \rightarrow$ $H_{2}(V, \partial V) \rightarrow H_{1}(\partial V) \rightarrow H_{1}(V)$ is exact, one has $r k_{\mathrm{z}}\left(H_{1}(V) / H_{1}(\partial V)\right)=\rho-$ $\sigma+\mu$. Similarly,

$$
H_{1}(X-V, \partial X-V) \rightarrow H_{0}(\partial X-V) \rightarrow H_{0}(X-V) \rightarrow 0
$$

is exact, and $r k_{\mathbf{Z}} H_{0}(\partial X-V)=\sigma, r k_{\mathbf{Z}} H_{0}(X-V)=1$, so

$$
\begin{aligned}
r k_{\mathbf{Z}} J & =r k_{\mathbf{z}} H_{1}(X-V, \partial X-V)-\sigma+1 \\
& =r k_{\mathbf{z}} H_{1}\left(S^{3}-V, \operatorname{Im} N\right)-\sigma+1 .
\end{aligned}
$$

Now each component of the link is the homology boundary of a (singular) surface in $S^{3}-V$, and so the natural map

$$
H_{1}(\operatorname{Im} N) \rightarrow H_{1}\left(S^{3}-V\right)
$$

is null. Therefore

$0-H_{1}\left(S^{3}-V\right) \rightarrow H_{1}\left(S^{3}-V, \operatorname{Im} N\right) \rightarrow H_{0}(\operatorname{Im} N) \rightarrow H_{0}\left(S^{3}-V\right) \rightarrow 0$ is exact, and so $r k_{\mathbf{z}} H_{1}\left(S^{3}-V, \operatorname{Im} N\right)=r k_{\mathbf{z}} H_{1}\left(S^{3}-V\right)+\mu-1=$ $r k_{\mathbf{z}} H_{1}(V)+\mu-1$ by Alexander duality $=\rho+\mu-1$. Thus $r k_{\mathbf{z}} J=\rho+\mu$ 
- $\sigma=r k_{\mathrm{z}}\left(H_{1}(V) / H_{1}(\partial V)\right)$, and so $\varepsilon_{0}(Q)$ is principal. This completes the proof of the theorem.

REMARKS. 1. Brown and Crowell asserted the somewhat more precise result (for $\mu=2$ ) that $A$ could be generated by 3 elements, of the form $\left(t_{1}-\right.$ 1) $p_{1}\left(t_{1}\right),\left(t_{2}-1\right) p_{2}\left(t_{2}\right)$ and $p_{1}\left(t_{1}\right)+p_{2}\left(t_{2}\right)-1$ where $p_{i}(1)=1$, and that the $i$ th longitude lay in $G^{\prime \prime}$ if and only if $p_{3-i}\left(t_{3-i}\right)$ were a unit [2]. This follows readily from $A=A_{1} \cap A_{2}$, where $A_{i}$ is the annihilator of the $i$ th longitude and equals $\left(t_{i}-1, p_{3-i}\left(t_{3-i}\right)\right)$ for some $p_{i}$, as above.

2. Fox and Smythe conjectured that if the longitudes were in $G^{\prime \prime}$, then the link would be a boundary link [6]. H. W. Lambert has constructed a 2-component homology boundary link which is not a boundary link, as a counterexample to this conjecture [4]. (Figure 1 of his paper is incorrectly drawn: the shorter longitude of this example does not map to 0 in the Alexander module (via Crowell's inclusion $0-G^{\prime} / G^{\prime \prime} \rightarrow A(G)$ [1]) and hence this link is not such a counterexample. $\left.{ }^{1}\right)$ Notice also that boundary links have the stronger (but less tractable?) property that the longitudes are in $\left(G_{\omega}\right)^{\prime}$ (where $G_{\omega}=\bigcap_{n=1}^{\infty} G_{n}$ is the intersection of the terms of the lower central series). This follows from the construction of the $\omega$-covering by splitting the link complement along Seifert surfaces, as in [3].

3. If $L$ is trivial then $\varepsilon_{\mu}(L)=\Lambda$, but the converse is false, even for knots ( $\mu=1$ ), for there exists nontrivial knots (for instance doubled knots with twist number 0 ) with Alexander polynomial 1 [5].

\section{REFERENCES}

1. R. H. Crowell, Corresponding group and module sequences, Nagoya Math. J. 19 (1961), $27-40$.

2. _ Private communication to N. F. Smythe, May 1976.

3. M. A. Gutierrez, Polynomial invariants of boundary links, Rev. Colombiana Mat. VIII (1974), 97-109.

4. H. W. Lambert, A 1-linked link whose longitudes lie in the second commutator subgroup, Trans. Amer. Math. Soc. 147 (1970), 261-269.

5. D. Rolfsen, Knots and links, Publish or Perish, Inc., Berkeley, California, 1976.

6. N. F. Smythe, Boundary links, Topology Seminar, Wisconsin, 1965, Ann. of Math. Studies, No. 60, Princeton Univ. Press, Princeton, N. J., 1966, pp. 69-72.

Department of Pure Mathematics, School of General Studies, Australian National University, Canberra, A. C. T. 2600, Australia

${ }^{1}$ Lambert has advised me that his argument is based on a slightly different figure. 